\title{
Conhecimento de Estudantes de Medicina de Diferentes Metodologias de Ensino sobre Manejo de Opioides em Doenças Crônicas
}

\section{Knowledge of Medical Students of Different Teaching Methodologies on Opioid Management in Chronic Diseases}

\section{TOSI, AB*; RODRIGUES,GG*; EBELING,B*; EUGÊNIO;C**}

* Discente de Medicina do Centro Universitário São Camilo, SP

** Docente do curso de Medicina Centro Universitário São Camilo - SP

\section{RESUMO:}

OBJETIVO: O presente estudo tem como objetivo principal avaliar alunos do 5 e 6ํㅡㅁ ano de medicina de Escolas Médicas do Estado de São Paulo em relação ao conhecimento sobre o manejo de opioides, afim de se avaliar se a limitação no uso de medicamentos opioides apresenta respaldo na formação acadêmica inadequada sobre o manejo de dor crônica. Ademais, verificaremos a possível influência de cada metodologia no aprendizado do aluno de graduação.

METODOLOGIA: Aplicação de questionário via Google Forms, com 18 questões de múltipla escolha, em estudantes do $5^{\circ}$ e $6^{\circ}$ ano de escolas médicas públicas e privadas do Estado de São Paulo, de metodologias ativa, mista e tradicional. Destas, 5 questões se referia a experiência do estudante ao longo da graduação e 8, os conhecimentos específicos sobre opioides. Realizou-se análise descritiva do perfil dos estudantes através de idade, semestre, graduação anterior, tipo de instituição e metodologia. Os conhecimentos específicos, foram categorizados em pouco, médio e bom conhecimento. A prevalência das categorias de desempenho foi comparada entre as diferentes metodologias de ensino pelo teste qui-quadrado, considerando-se um nível de significância de valor de $p<0,05$. Trabalho previamente aprovado pelo Comitê de Ética (CAAE:37763320.7.0000.0062) 
RESULTADOS: 169 estudantes de Medicina participaram do estudo. Destes, $72 \%$ da metodologia tradicional, $75 \%$ da mista e $70 \%$ da ativa, afirmaram apresentar aula sobre manejo de opioides durante a graduação. Acerca da Escala Visual da Dor, 97\% da metodologia tradicional, $98 \%$ da mista e $96 \%$ da ativa afirmaram conhecê-la. $43 \%$ dos alunos da metodologia tradicional, $45 \%$ da mista e $23 \%$ da tradicional relataram falta de preparo para a prescrição de opioides. $55 \%$ da metodologia tradicional, $62,5 \%$ da mista e

$53 \%$ da ativa relataram a experiência de preencher receituário amarelo durante a

graduação. Sobre a categorização dos acertos, 51\% dos alunos da metodologia ativa obtiveram pouco conhecimento, $69 \%$ da mista e $64 \%$ da tradicional obtiveram médio conhecimento.

CONCLUSÃO: O manejo inadequado de opioides na dor crônica apresenta respaldo na formação acadêmica de estudantes de Medicina. Com isso, mostra-se necessário o aperfeiçoamento do conteúdo na graduação, a fim de proporcionar melhor tratamento aos pacientes.

DESCRITORES: Manejo opioide, conhecimento prescrição

\section{ABSTRACT}

OBJECTIVE: The main objective of the present study is to evaluate 5th and 6th year medical students at Medical Schools of the State of São Paulo in relation to their knowledge about opioid management, in order to assess whether the limitation in the use of opioid drugs is supported. in inadequate academic training on the management of chronic pain. Furthermore, we will verify the possible influence of each methodology on undergraduate student learning.

METHODOLOGY: A questionnaire was applied via Google Forms, with 18 multiple-choice questions, to 5th and 6th grade students from public and private medical schools in the State 
54 of São Paulo, using active, mixed and traditional methodologies. Of these, 5 questions referred to the student's experience during graduation and 8 referred to specific knowledge about opioids. Descriptive analysis of the profile of students was carried out according to age, semester, previous graduation, type of institution and methodology. Specific knowledge was categorized into little, medium and good knowledge. The prevalence of performance categories was compared between the different teaching methodologies using the chi-square test, considering a significance level of $p$ value $<0.05$. Work previously approved by the Ethics Committee (CAAE: 37763320.7.0000.0062)

RESULTS: 169 medical students participated in the study. Of these, $72 \%$ of the traditional methodology, $75 \%$ of the mixed and $70 \%$ of the active, claimed to present a class on opioid management during graduation. Regarding the Visual Pain Scale, $97 \%$ of the traditional methodology, $98 \%$ of the mixed and $96 \%$ of the active ones claimed to know it. $43 \%$ of the students of the traditional methodology, $45 \%$ of the mixed and $23 \%$ of the traditional ones reported lack of preparation for the prescription of opioids. $55 \%$ of the traditional methodology, $62.5 \%$ of the mixed and $53 \%$ of the active ones reported the experience of filling out yellow prescriptions during graduation. On the categorization of correct answers, $51 \%$ of the active methodology students had little knowledge, $69 \%$ of the mixed and $64 \%$ of the traditional ones had medium knowledge.

CONCLUSION: Inadequate management of opioids in chronic pain has support in the academic education of medical students. Thus, it is necessary to improve the content in graduation, in order to provide better treatment for patients.

DESCRIPTORS: Opioid management, prescription knowledge

\section{INTRODUÇÃO}


A dor crônica acomete parcela significativa da população brasileira e sua manifestação, em adultos, compromete a capacidade física e funcional do indivíduo, diminuindo sua qualidade de vida. $(1,2)$

No caso do câncer, a dor moderada ou intensa está presente em 30\% dos indivíduos já em tratamento e sua prevalência aumenta com a progressão da doença, chegando a acometer 50 a $75 \%$ dos pacientes(3)

Segundo PIMENTA, et. al (2006), diversos pacientes que sofrem de dor crônica apresentam alívio insatisfatório após o tratamento. Esse fato está diretamente relacionados com a capacitação dos profissionais atuantes na área, os quais desconhecem métodos preconizados para avaliar a dor, bem como, apresentam receios e falta de capacitação para o uso de opioides no manejo dos processos dolorosos $(4,5)$

Atualmente, as opções de analgesia incluem os anti-inflamatórios não esteroidais, acetaminofeno, opioides fracos e fortes, relaxantes musculares, anticonvulsivantes e antidepressivos, sendo que a maioria desses é adequada para tratar a dor de leve a moderada em curto prazo.(6,7) Para a dor de moderada a grave, os opioides fortes são geralmente a primeira escolha $(6-8)$

A administração de opioides sem o conhecimento, tanto do indivíduo, quanto do profissional de saúde, tem o potencial de resultar em administração incorreta, podendo trazer prejuízos ao paciente.

Um estudo feito por Daudt et al, no qual foram revisadas 1107 prescrições relativas a 445 pacientes com dor internados, mostrou que apenas $6,5 \%$ das prescrições foram consideradas adequadas, concluindo em seu estudo que, o manejo inadequado da dor por parte dos profissionais de saúde estava presente. (9)

Diversas hipóteses foram levantadas para explicar a prescrição incorreta de opioides, tanto em casos de neoplasias, como em situações mais agudas, por exemplo no pós- 
operatório. Algumas das principais encontradas na literatura, são: dificuldade da equipe médica para avaliar corretamente a dor sentida pelo paciente; deficiências no ensino médico a respeito do uso de opioides e do tratamento da dor; o medo, por parte do médico e da equipe, do desenvolvimento de dependência física e psíquica do paciente; e as dificuldades legais para se prescrever tais drogas encontradas em alguns países (9-11).

Com isso pode-se observar que o uso de opioides abrange fatores sociais, econômicos, legais, educacionais, éticos, políticos entre outros.

Em outro estudo, Kulkamp et al avaliou a percepção e conhecimento de profissionais da saúde a respeito do tema dor, além de aspectos abordados na utilização de medicamento opioides, como legislação e efeitos colaterais. Também foram detectados aspectos subjetivos relacionados ao uso de medicamentos opioides, como crenças, indícios de medo e preconceito (12)

Observa-se, a partir desses estudos, que há anos existe um déficit no conhecimento dos profissionais da saúde em relação a prescrição adequada de opioides no manejo da dor, que varia desde a sub prescrição até a prescrição de forma ilimitada e sem critérios, levando a casos de sobreprescrição $(13,14)$

A partir deste cenário, hoje ainda existente em relação ao uso de opioides no manejo da dor crônica, o presente trabalho visa avaliar alunos do $5^{\circ}$ e $6^{\circ}$ ano de medicina de Escolas Médicas do Estado de São Paulo, com o objetivo de verificar se durante a formação acadêmica é fornecido conhecimento adequado aos alunos sobre o manejo correto de opioides na dor crônica.

Além disso, tendo em vista que, atualmente, as escolas médicas apresentam diferentes metodologias de ensino (tradicional, mista e ativa) realizaremos uma comparação entre elas, de acordo com as respostas obtidas. 
Com isso, poderemos avaliar se a limitação no uso de medicamentos opioides apresenta respaldo na formação acadêmica inadequada sobre o manejo de dor crônica. Ademais, verificaremos a possível influência de cada metodologia no aprendizado do aluno de 131 graduação.

\section{MÉTODO}

\subsection{Participantes}

opioides, um questionário contendo 18 questões de múltipla escolha foi criado utilizando

151 ferramentas online para a sua aplicação (Google Forms). Destas, 5 questões eram

152 referentes a experiência do estudante ao longo da graduação sobre a prescrição de 
opioides (questões 6 a 10) e 8, referentes a conhecimentos específicos sobre opioides (questões 11 a 18).

O questionário (Anexo 2) foi enviado através de redes sociais para o público alvo.

\subsection{Análise dos dados}

A avaliação do perfil dos estudantes que responderam ao questionário foi realizada através de análise descritiva de seus dados pessoais (idade), semestre que está cursando, graduação anterior, tipo de instituição que frequenta (pública ou privada) e a metodologia de ensino da faculdade (metodologia tradicional, mista ou ativa).

Os resultados obtidos através das perguntas sobre o conhecimento de opioides em cada grupo foram expressos em valores absolutos e percentuais (i.e. em relação ao total de indivíduos de cada grupo).

O conhecimento individual sobre o manejo adequado da dor foi realizado através da categorização dos indivíduos em: pouco conhecimento (0-3 acertos), médio conhecimento (4-6 acertos) e bom conhecimento (7-8 acertos). Para isso, foram consideradas as respostas das perguntas de número 11 a 18 , sendo os resultados obtidos expressos em valores percentuais e relacionados a metodologia de ensino prevalente em cada categoria. A prevalência das categorias de desempenho (i.e. pouco conhecimento, médio conhecimento e bom conhecimento) foi comparada entre as diferentes metodologias de ensino pelo teste qui-quadrado, considerando-se um nível de significância de valor de $p<$ 0,05. Com testes post-hoc, foram calculados os resíduos padronizados ajustados para permitir a identificação dos valores estatisticamente significantes (i.e. valor correspondente de $p<0.006$ ). Essas análises foram realizadas de acordo com os procedimentos estabelecidos por Beasley e Schumacker (15), utilizando o programa Statisctical Package for the Social Sciences (SPSS). 
3. RESULTADOS

\subsection{Amostra}

169 estudantes de medicina responderam ao Termo de Consentimento Livre e

182 Esclarecido e participaram do estudo. As características da amostra estão representadas

183 na Tabela 1

184

Tabela 1. Características da amostra.

\begin{tabular}{|c|c|c|c|}
\hline \multirow{2}{*}{$\begin{array}{l}\text { Características } \\
\text { Metodologia }\end{array}$} & Metodologia & \multicolumn{2}{|l|}{ Metodologia } \\
\hline & $\begin{array}{l}\text { Tradicional } \\
\quad(n=58)\end{array}$ & $\begin{array}{l}\text { Mista } \\
(n=64)\end{array}$ & $\begin{array}{l}\text { Ativa } \\
(n=47)\end{array}$ \\
\hline \multicolumn{4}{|l|}{ Idade } \\
\hline $18-25$ anos (117) & 37 (64\%) & 38 (59\%) & 37 (89\%) \\
\hline $26-30$ anos (39) & $14(24 \%)$ & 18 (28\%) & $7(15 \%)$ \\
\hline $31-35$ anos (10) & $1(1 \%)$ & $6(9 \%)$ & $3(6 \%)$ \\
\hline$>35$ anos (3) & $1(1 \%)$ & $2(3 \%)$ & $0(0 \%)$ \\
\hline \multicolumn{4}{|l|}{ Semestre } \\
\hline 9o semestre (50) & 17 (29\%) & $11(17 \%)$ & $17(36 \%)$ \\
\hline $10^{\circ}$ semestre (13) & $0(0 \%)$ & $6(9 \%)$ & $8(17 \%)$ \\
\hline $11^{\circ}$ semestre (42) & $12(21 \%)$ & $21(33 \%)$ & $12(26 \%)$ \\
\hline $12^{\circ}$ semestre (64) & $29(50 \%)$ & $25(39 \%)$ & $10(21 \%)$ \\
\hline \multicolumn{4}{|c|}{ Graduação anterior } \\
\hline $\operatorname{Sim}(15)$ & $2(3 \%)$ & $8(13 \%)$ & $5(11 \%)$ \\
\hline Não (154) & $56(97 \%)$ & $56(87 \%)$ & $42(89 \%)$ \\
\hline \multicolumn{4}{|c|}{ Tipo de instituição } \\
\hline Pública (45) & $23(40 \%)$ & $7(11 \%)$ & $15(32 \%)$ \\
\hline Privada (124) & $35(60 \%)$ & 57 (89\%) & $32(68 \%)$ \\
\hline
\end{tabular}

\subsection{Questionário}

Dos alunos que responderam ao questionário, 72\% da metodologia tradicional, 75\%

188 da metodologia mista e $70 \%$ da metodologia ativa, afirmaram apresentar aula sobre manejo de opioides durante a graduação. 
191 tradicional, 98\% da metodologia mista e 96\% da metodologia ativa responderam conhecer

192 a escala visual da dor.

$19343 \%$ dos alunos da metodologia tradicional, $45 \%$ da metodologia mista e $23 \%$ da 194 metodologia ativa, relataram não se sentir preparado para a prescrição de opioides.

Quanto a experiência de ter preenchido um receituário amarelo durante a graduação, 55\% dos alunos de metodologia tradicional, $62,5 \%$ da metodologia mista e $53 \%$ da metodologia ativa relataram ter tido a experiencia de preencher receituário amarelo 198 durante a graduação.

A avaliação global ( $n^{\circ}$ absoluto e percentual) do conhecimento sobre manejo de opioides, sem a comparação em relação a metodologia de ensino foi expressa na Tabela 2

Os valores percentuais e absolutos referentes as respostas das perguntas específicas sobre o conhecimento de opioides de acordo com a metodologia de ensino 203 foram descritos na Tabela 3.

Tabela 2. Valores percentuais e absolutos referentes as respostas das perguntas específicas sobre o conhecimento de opioides (Questões 11 a 18) em relação ao no absoluto de alunos participantes da pesquisa

\section{Questão}

Amostra $(n=169)$

11) A morfina é o opioide mais potente para uso domiciliar?

Resposta correta (sim)

Resposta incorreta (não, não sei)

12) A dose de morfina via oral é a mesma em relação a dose endovenosa?

Resposta correta (Não)

Resposta incorreta (sim, não sei)

13) É comum e importante a associação de mais

um opioide na prescrição de um paciente com dor forte? 
Resposta correta (Não)

Resposta incorreta (sim, não sei)

14) A via subcutânea deve ser evitada para uso

de opioides de apresentação endovenosa?

Resposta correta (não)

Resposta incorreta (sim, não sei)

$31(18 \%)$

$128(75 \%)$

15) Paciente que nunca usou opioide, chega ao consultório com neoplasia de mama metastática para ossos, deve-se iniciar o tratamento da dor respeitando a ordem dos degraus da escala de analgesia, iniciando-se por opioide fraco?

Resposta correta (não)

Resposta incorreta (sim, não sei)

$84(50 \%)$

$85(50 \%)$

16) Paciente que usava morfina $10 \mathrm{mg}$ de $4 / 4$ horas

em casa vem ao pronto socorro por piora da dor.

Além de investigar a possível causa da piora o médico

deverá prescrever tramadol endovenoso?

Resposta correta (não)

Resposta incorreta (sim, não sei)

$93(55 \%)$

$76(44 \%)$

17) O principal efeito adverso dos opioides é obstipação?

Resposta correta (sim)

Resposta incorreta (não, não sei)

$104(62 \%)$

$65(38 \%)$

18) O uso de opioides por via transdérmica (adesivo) é uma excelente opção de tratamento para dor crônica ainda não bem controlada?

Resposta correta (não)

Resposta incorreta (sim, não sei)
$39(23 \%)$

$130(77 \%)$

Tabela 3. Valores percentuais e absolutos referentes as respostas das perguntas específicas sobre o conhecimento de opioides (Questões 11 a 18)

\begin{tabular}{|c|c|c|c|}
\hline \multirow[t]{2}{*}{ Questão } & \multirow[b]{2}{*}{$\begin{array}{l}\text { Tradicional } \\
\quad(n=58)\end{array}$} & \multicolumn{2}{|c|}{ Metodologia } \\
\hline & & $\begin{array}{l}\text { Mista } \\
(n=64)\end{array}$ & $\begin{array}{c}\text { Ativa } \\
(\mathrm{n}=47)\end{array}$ \\
\hline \multicolumn{4}{|c|}{$\begin{array}{l}\text { 11) A morfina é o opioide mais potente para uso } \\
\text { domiciliar? }\end{array}$} \\
\hline $\operatorname{Sim}(52)$ & $16(28 \%)$ & $18(28 \%)$ & $18(38 \%)$ \\
\hline Não (91) * & 31 (53\%) & $39(61 \%)$ & $21(45 \%)$ \\
\hline Não sei (26) & 11 (19\%) & $9(11 \%)$ & $8(17 \%)$ \\
\hline \multicolumn{4}{|c|}{$\begin{array}{l}\text { 12) A dose de morfina via oral é a mesma em } \\
\text { relação a dose endovenosa? }\end{array}$} \\
\hline $\operatorname{Sim}(7)$ & $1(2 \%)$ & $4(6 \%)$ & $2(4 \%)$ \\
\hline Não $(147)^{*}$ & $55(95 \%)$ & $56(88 \%)$ & $36(77 \%)$ \\
\hline
\end{tabular}


13) É comum e importante a associação de mais um opioide na prescrição de um paciente com dor forte?

Sim (44)

Não $(100)^{*}$ $14(24 \%)$ $35(60 \%)$ $19(30 \%)$ $38(59 \%)$ Não sei (25) $9(16 \%)$ $7(15 \%)$

$11(23 \%)$ $27(57 \%)$ $9(19 \%)$

14) A via subcutânea deve ser evitada para uso de opioides de apresentação endovenosa?
Sim (78)
Não $(31)^{*}$
$24(41 \%)$
$30(47 \%)$
$24(51 \%)$
$15(23 \%)$
$6(13 \%)$
Não sei (60)
$10(17 \%)$
$19(30 \%)$
$17(36 \%)$

15) Paciente que nunca usou opioide, chega ao consultório com neoplasia de mama metastática para ossos, deve-se iniciar o tratamento da dor respeitando a ordem dos degraus da escala de analgesia, iniciando-se por opioide fraco?
Sim (73)
$29(50 \%)$
$25(39 \%)$
$19(40 \%)$
Não $(84)^{*}$
$27(47 \%)$
Não sei (12)
$29(50 \%)$
$34(53 \%)$
$23(49 \%)$
$25(39 \%)$
$19(40 \%)$

16) Paciente que usava morfina $10 \mathrm{mg}$ de $4 / 4$ horas em casa vem ao pronto socorro por piora da dor.

Além de investigar a possível causa da piora o médico deverá prescrever tramadol endovenoso?

$\begin{array}{lrrr}\text { Sim }(43) & 12(21 \%) & 12(19 \%) & 9(40 \%) \\ \text { Não (93)* } & 35(60 \%) & 42(66 \%) & 16(34 \%) \\ \text { Não sei (33) } & 11(19 \%) & 10(16 \%) & 12(26 \%)\end{array}$

17) O principal efeito adverso dos opioides é obstipação?
$\operatorname{Sim}(104)^{*}$
Não (48)
$32(55 \%)$
$18(31 \%)$
$48(75 \%)$
$24(51 \%)$
Não sei (17)
$8(14 \%)$
$13(20 \%$
$17(36 \%)$
$6(13 \%)$

18) O uso de opioides por via transdérmica (adesivo) é uma excelente opção de tratamento para dor crônica ainda não bem controlada?
Sim (78)
$25(43 \%)$
$35(55 \%)$
$18(38 \%)$
Não (39)*
$11(19 \%)$
$12(19 \%)$
16 (34\%)
Não sei (52)
$17(29 \%)$
17 (27\%)
$18(38 \%)$ 
O conhecimento dos alunos foi categorizado em: pouco conhecimento, médio conhecimento e bom conhecimento, de acordo com o número de acertos nas perguntas

213 específicas sobre opioides (questões 11 a 18). Os dados obtidos encontram-se na Tabela 2143.

Tabela 2. Categorização dos acertos obtidos pelos participantes.

\begin{tabular}{|c|c|c|c|c|}
\hline & \multicolumn{2}{|c|}{ Desempenho } & \multicolumn{2}{|c|}{ Teste Qui-Quadrado } \\
\hline Pouco & Médio & Bom & Total & \\
\hline n (\%) $24(51 \%)$ & $22(47 \%)$ & $1(2 \%)$ & $47(100 \%)$ & \\
\hline Resíduos ajustados $2,85^{\star \star}$ & $-2,34$ & $-0,99$ & & \\
\hline n (\%) $15(23 \%)$ & $44(69 \%)$ & $5(8 \%)$ & $64(100 \%)$ & $p=0,033^{*}$ \\
\hline Resíduos ajustados $\quad-2,33$ & 1,62 & 1,47 & & \\
\hline Tradicional n (\%) $19(33 \%)$ & $37(64 \%)$ & $2(3 \%)$ & $58(100 \%)$ & \\
\hline Resíduos ajustados & 0,55 & $-0,57$ & & \\
\hline
\end{tabular}

${ }^{*}$ valor com significância estatística no teste qui-quadrado.

**valores de resíduos ajustados com significância estatística.

diferentes metodologias de ensino sobre o manejo correto de opioides nas doenças crônicas.

\section{DISCUSSÃO}

O presente estudo buscou avaliar o conhecimento dos estudantes de medicina de ativa apresentou desempenho pior em relação a metodologia tradicional e mista $(p=0,033)$.

As principais dificuldades encontradas pelos alunos referem-se ao conhecimento sobre

227 a potência dos opioides, as possíveis associações farmacológicas, as principais vias de 228 administração disponíveis e a utilização correta da escala de analgesia. 
229 Percebeu-se que o conhecimento sobre a dose de administração da morfina e o 230 principal efeito adverso dos opioides está consolidado.

231 Atualmente, poucos estudos encontrados na literatura avaliaram o conhecimento de 232 médicos e profissionais da saúde sobre a prescrição adequada de opioides. Os estudos 233 mais recentes abordam a prescrição ilimitada e ao uso abusivo dessas substâncias. $234(13,14)$

235 Devulder. J (1999) avaliou 45 médicos e 28 enfermeiros que tratavam de pacientes 236 terminais com câncer, através de questionário contendo perguntas sobre o uso clínico, 237 efeitos esperados e efeitos colaterais do uso da morfina. O resultado encontrado mostrou 238 que a maioria dos profissionais (93\%) demonstrou desejo de ser melhor treinado para cuidado paliativo, 37\% não sabiam que não havia limite de dose para morfina, $26 \%$ 240 conheciam o risco de depressão respiratória, 99\% conheciam corretamente a via de 241 administração da morfina, 21\% sabiam tratar corretamente dor neuropática e 41\% dor 242 nociceptiva. (16)

$243 \quad$ Este estudo se assemelha com os resultados obtidos em nossa pesquisa, mostrando 244 que os profissionais da saúde conhecem adequadamente a via de administração da 245 morfina, diferindo esse conhecimento em relação a via de administração de outros 246 opioides.

247 Outro estudo realizado por Pflughaupt,M (2010) verificou que médicos apresentavam 248 pouco conhecimento sobre o uso da Escala analgésica da OMS, o que compactuou com 249 os resultados obtidos no nosso estudo, o qual mostrou que apenas metade da amostra 250 tinha conhecimento sobre a escala analgésica e sua aplicação.(17) 251 Nossos resultados demonstraram que a prescrição inadequada de opioides, ainda 252 existente na área da saúde, apresenta respaldo na formação acadêmica. 
Observamos uma diferença no desempenho em relação as diferentes metodologias de ensino, tendo a metodologia tradicional e a mista apresentado maior conhecimento, quando comparadas com a metodologia ativa. Tal diferença poderia ser explicada, devido ao fato de a maior parte da amostra da metodologia ativa se encontrar ainda no 9응 semestre (36\%), não apresentando a vivência prática de prescrições durante o internato.

Para uma comparação mais adequada entre as metodologias, um estudo com uma 273 fim de melhorar o tratamento oferecido ao pacientes

\section{CONCLUSÕES}

O manejo inadequado de opioide na dor crônica, ainda encontrado na prática clínica, apresenta respaldo na formação acadêmica de estudantes de Medicina. É necessário que a educação dos mesmos em relação a prescrição de opioides, seja melhor aperfeiçoada, a

\section{REFERÊNCIAS}

1. Pessini L, Saunders DC, Cassel E. Humanização da dor e sofrimento humanos no contexto hospitalar. Rev Bioet. 2002;16-7. 
278 2. CP Olinto, MP Oliveira, MHMZuardi NB. Grupos educativos no manejo de pacientes 279 com dor crônica. Prática Hosp. 2005;41.

280 3. GL Salamonde, N Verçosa, L Barrucand AC. Clinical and therapeutic analysis of

oncology patients treated at the pain and palliative care program of the Hospital Universitário Clementino Braga Filho in 2003. Rev Bras Anestesiol. 2006;56(6):60218.

4. Pimenta, C. A. de M., Mota, D. D. C. de F., \& Cruz D de ALM da. Dor e cuidados paliativos: enfermagem, medicina e psicologia. Manole. 2006;

5. Kipel A. AO USO DE ANALGÉSICOS OPIÁCEOS. Red Rev Cient América Lat y el Caribe, España y Port. 2004;13:303-8.

6. Coluzzi F, Taylor R, Pergolizzi J V, Mattia C, Raffa RB. Orientação para boa prática clínica para opioides no tratamento da dor: os três "Ts" - titulação (teste), ajustes (individualização), transição (redução gradual). Rev Bras Anestesiol. $2016 ; 66(3): 310-7$.

7. Kraychete DC, Sakata RK. Uso e Rotação de Opioides para Dor Crônica não Oncológica. Rev Bras Anestesiol. 2012;62:558-62.

8. Saúde S de. Diretriz de tratamento farmacológico da dor nos pronto atendimentos. 2018;

9. A.W. DAUDT, E. HADLICH, M.A. FACIN, R.M.S. APRATO RPP. Opióides no manejo da dor - uso correto ou subestimado ? Dados de um hospital universitário. Rev Ass Med Bras. 1998;44(2):106-10.

10. Hammack JE LC. Use of orally administred opioids for cancer related pain. Mayo Clin Proc [Internet]. 1994;69:384-90. Available from:

https://www.mayoclinicproceedings.org/article/S0025-6196(12)62226-5/fulltext

11. M M. Alivio da dor no câncer. Atheneu. 1992; 
12. Kulkamp IC, Barbosa CG, Bianchini KC. The perception of health professionals about pain management and opioid use : a qualitative study. Cien Saude Colet. 2008;13(Sup):72:721-31.

13. Kaye AD, Jones MR, Kaye AM, Ripoll JG, Galan V, Beakley BD, et al. Prescription Opioid Abuse in Chronic Pain: An Updated Review of Opioid Abuse Predictors and Strategies to Curb Opioid Abuse: Part 1. Pain Physician. 2017;20:93-110.

14. Clark DJ, Schumacher MA. America's Opioid Epidemic: Supply and Demand Considerations. Spec Artic. 2017;125(5):1667-74.

15. Beasley TM. Multiple Regression Approach to Analyzing Contingency Tables : Post Hoc and Planned Comparison Procedures. J Exp Educ. 2014;64(October 1995):79_ 93.

16. Devulder J. Persisting misconceptions of belgian physicians and nurses about cancer pain treatment. Acta Clin Belg. 1999;54(6):46-50.

17. Pflughaupt M. RSGGUKTKRS. Physicians' knowledge and attitudes concerning the use of opioids in the treatment of chronic cancer and non-cancer pain. Zusammenfassung. 2010;267-75.

18. Nascimento, LA ; Kreling M. Avaliação da dor como quinto sinal vital : opinião de profissionais de enfermagem *. Acta Paul Enferm. 2011;24(1):50-4.

19. Fonseca, A; Geobanini F. Cuidados Paliativos na Formação do Profissional da Área de Saúde. Rev Bras Educ Med. 2011;37(1):120-5.

\section{ANEXOS}

\section{Anexo 1 - Termo de Consentimento Livre e Esclarecido}


327 Título da pesquisa: Conhecimento de estudantes de medicina de diferentes metodologias 328 de ensino sobre manejo de opioides em doenças crônicas Prezado (a) Aluno (a) Esta 329 pesquisa está sendo desenvolvida por Amanda Barboza Tosi, Bettina Ebeling, Giovanna 330 Gonzalez Rodrigues, alunas do Curso de Medicina do Centro Universitario São Camilo, sob 331 a orientação da Cecilia Eugênio, professora responsável pela disciplina eletiva de Cuidados 332 Paliativos. O objetivo do estudo é avaliar o conhecimento dos estudantes de medicina sobre 333 o manejo adequado de opioides. A finalidade é verificar se durante a formação acadêmica é 334 fornecido conhecimento adequado aos alunos sobre a prescrição de opioides na dor crônica 335 Com isso poderemos avaliar se há algum respaldo na formação acadêmica que justifique a 336 limitação na utilização de medicamento opioides e verificar se há a necessidade de ampliar o conhecimento sobre os mesmos durante a graduação. Para a participação no estudo é necessá rio o preenchimento do questionário abaixo que contém 18 questões de múltipla escolha, como também a sua autorização para apresentar os resultados deste 13 estudo em eventos da área da saúde e/ou publicar em revistas cientifica nacional e/ou internacional. Por ocasião da publicação dos resultados seu nome será mantido em sigilo absoluto. 342 Esclarecemos que sua participação no estudo é voluntária e, portanto, a desistência da participação pode ser realizada a qualquer momento após o início do questionário. Os pesquisadores estarão a sua disposição para qualquer esclarecimento que considere necessário em qualquer etapa da pesquisa. Considerando, que fui informado(a) dos objetivos e da relevância do estudo proposto e de como será minha participação, declaro o meu consentimento em participar da pesquisa como também concordo que os dados obtidos na investigação sejam utilizados para fins científicos.

Assinatura do participante. 
353 Caso necessite de maiores informações sobre o presente estudo, favor entrar em contato 354 através do seguinte email: xxxxxxxxxx@gmail.com

356 Anexo II - Questionário

\section{Questionário respondido por estudantes de medicina do 6ano}

1) Idade

$\square$ 18- 25 anos

$\square$ 26-30 anos

$\square$ 31-35 anos

$\square>35$ anos

2) Qual semestre está cursando?

$\square 11^{\circ}$ semestre

$\square 12^{\circ}$ semestre

3) Possui outra graduação?

$\square \operatorname{Sim}$

$\square$ Não

4) Estuda em qual tipo de instituição?

$\square$ Pública

$\square$ Privada

5) Qual o método de sua faculdade?

$\square$ Tradicional 
$\square$ Misto (Metodologia ativa + Tradicional

$\square$ Metodologia ativa

6) Você teve aula sobre manejo de opioides durante a graduação?

$\square \operatorname{Sim}$

$\square$ Não

7) Você conhece a Escala Visual de dor?

$\square \operatorname{Sim}$

$\square$ Não

8) Você se sente preparada(o) para prescrever opioide?

$\square \operatorname{Sim}$

$\square$ Não

9) Você alguma vez já preencheu um receituário amarelo?

$\square \operatorname{Sim}$

$\square$ Não

10) Você já cuidou de algum paciente que usava Metadona?

$\square \mathrm{Sim}$

$\square$ Não

11) A morfina é o opioide mais potente para uso domiciliar?

$\square \mathrm{Sim}$ 
$\square$ Não

$\square$ Não sei

12) A dose da morfina via oral é a mesma em relação a sua dose endovenosa?

$\square \operatorname{Sim}$

$\square$ Não

$\square$ Não sei

13) É comum e importante a associação de mais um opioide na prescrição de um paciente com dor forte?

$\square \operatorname{Sim}$

$\square$ Não

$\square$ Não sei

14) A via subcutânea deve ser evitada para uso de opioide de apresentação endovenosa?

$\square \operatorname{Sim}$

$\square$ Não

$\square$ Não sei

15) Paciente que nunca usou opioide, chega ao consultório com neoplasia de mama metastática para ossos, deve-se iniciar o tratamento da dor respeitando a ordem dos degraus da escala de analgesia, iniciando-se por opioide fraco?

$\square \operatorname{Sim}$

$\square$ Não 
$\square$ Não sei

16)Paciente que usava morfina $10 \mathrm{mg}$ de $4 / 4$ horas em casa vem ao pronto socorro por piora da dor. Além de investigar a possível causa da piora o médico deverá prescrever tramadol endovenoso?

$\square \operatorname{Sim}$

$\square$ Não

$\square$ Não sei

17) O principal efeito adverso dos opioides é a obstipação?

$\square \operatorname{Sim}$

$\square$ Não

$\square$ Não sei

18) O uso de opioides por via transdérmica (adesivos) é uma excelente opção de tratamento para dor crônica ainda não bem controlada?

$\square \mathrm{Sim}$

$\square$ Não

$\square$ Não sei 